\title{
Estrés: una propuesta naturalista
}

\section{Stress: a naturalistic proposal}

\author{
María de Lourdes Rodríguez Campuzano ${ }^{1}$ \\ FES UNAM Iztacala, Ciudad de México
}

(Recepción: Febrero 2010 - Aceptación: Julio 2010)

\section{Resumen}

En este texto se revisan algunos de los aspectos planteados en el estudio del estrés, particularmente, desde la concepción de Lazarus y Folkman (1991). Se sostiene que esta aproximación es dualista y que las investigaciones llevadas a cabo desde esta perspectiva emplean criterios morfológicos que no han permitido dar cuenta de aspectos importantes de este fenómeno. Con base en una perspectiva interconductual, se propone tomar en cuenta tres criterios para estudiar este tipo de comportamiento: la naturaleza funcional de la situación, los niveles de aptitud funcional del comportamiento y sus diferentes dimensiones. Se enfatiza el carácter individual de las reacciones de estrés y se sugiere que a partir de los criterios propuestos se elabore una taxonomía de situaciones genéricas para estudiar dichas reacciones, como parte de patrones comportamentales complejos.
\end{abstract}

Palabras claves: estrés, afrontamiento, criterios morfológicos, criterios funcionales, dimensiones del comportamiento.

\begin{abstract}
Some of the stress related topics, especially from the conceptual framework of Lazarus and Folkman are reviewed on this work. It is sustained that this approach is dualistic and that the research made from this view is made on the basis of morphological criteria that don't allow studying important elements of this kind of behavior. From an interbehavioral approach three functional criteria are proposed to study this phenomenon: the functional nature of situations, aptitude levels of behavior, and its three dimensions. Emphasis is made on the singular and individual nature of stress reactions. Finally it is suggested to take into account these functional criteria to develop a generic situational taxonomy to study these reactions as parts of complex behavioral patterns.
\end{abstract}

Key words: stress, coping, morphological criteria, functional criteria, behavioral dimensions.

1 Correspondencia a: María de Lourdes Rodríguez Campuzano. Pekín 6, Jardines de Bellavista, Tlalnepantla, Estado de México, C.P. 54054. Teléfono: (0155) 536154 01. E-mail: carmayu5@yahoo.com. 
El estrés es uno de los aspectos que actualmente se considera fundamental para la explicación de una gran cantidad de enfermedades. La psicología ha abordado el tema de distintas maneras, a partir de que Hans Selye (1936) definió el concepto como un Síndrome General de Adaptación constituido por una respuesta general del organismo ante cualquier estímulo estresor o situación estresante.

Actualmente se reconocen tres perspectivas del estrés. La primera de ellas, aborda al estrés como estímulo, supone que el estrés es algo que ocurre en el ambiente y que está constituido por una demanda para la persona; muchos autores llaman a esto fuente de estrés o estresores y quienes los investigan se dedican a clasificarlos y medirlos. En la segunda perspectiva, se considera al estrés como respuesta, su punto de partida son los diversos cambios, ya sean de tipo conductual o biológico, que presentan las personas como consecuencia de un conjunto particular de demandas. Finalmente, la más reciente es la perspectiva del estrés como transacción entre la persona y su medio, en donde subyace una concepción cognoscitiva que plantea al estrés como un proceso (Cassidy, 1999).

Esta última perspectiva es la que ejerce mayor influencia en la investigación en tiempos recientes. Por tal razón, en este trabajo se presenta una concepción alternativa del fenómeno y se propone el desarrollo de un instrumento como una aproximación inicial de investigación del mismo.

En la concepción cognoscitiva o transaccional, Lazarus y Folkman (1986) aseguran que el estrés hace referencia a una relación existente entre persona y entorno y que la clave de esta transacción es la valoración personal de la situación. Rodríguez (1995) se refiere a la cantidad de recursos que el estresor parece requerir, cuando el ajuste entre las demandas de la situación y sus recursos no es adecuado, entonces se produce una discrepancia que puede ser real o percibida. A la valoración de esta discrepancia Lazarus y Folkman (1986) la llaman evaluación cognitiva, y comentan que es un proceso mental mediante el cual se evalúan dos factores: si las exigencias de la situación amenazan el bienestar del individuo y los recursos disponibles para responder a estas demandas.

Para Rodríguez (1995) existen básicamente dos tipos de factores que influyen en la evaluación de un acontecimiento como estresante: a) los factores personales que incluyen elementos cognitivos, motivacionales, de personalidad y hábitos comportamentales, y b) los factores situacionales. Entre los elementos cognitivos, Lazarus y Folkman (1986) destacan las creencias y los compromisos. Las creencias se consideran nociones pre-existentes acerca de la realidad, son configuraciones cognitivas moldeadas social y culturalmente. Los compromisos revelan lo que es importante para la persona y lo que tiene significado para ella. Los factores situacionales incluyen las características distintivas objetivas del acontecimiento, pueden ser de pérdida (la remoción de una fuente deseada de refuerzo positivo) o de castigo (ocurrencia de una situación aversiva); y las dimensiones objetivas de la situación entre las que se encuentran: valencia de la situación (su potencialidad estresante inherente), su controlabilidad (las oportunidades de control inherentes a la situación), su mutabilidad (la probabilidad de que la situación cambie por si misma), su ambigüedad (el grado en el que una situación carece por si misma de suficiente información como para que el individuo pueda hacerse una idea clara de ella), su potencial de ocurrencia o recurrencia (la capacidad de ocurrir o volver a ocurrir inherente a la situación), y su momento o cronología respecto al ciclo vital (momento de la vida de una persona en que ocurre el acontecimiento).

Desde esta perspectiva se considera que hay tres clases de valoración de las situaciones: la primaria, cuando el individuo se enfrenta por primera vez a una situación y evalúa los efectos de dicha situación sobre su propio bienestar (Brannon y Feist, 2001). Después de que una persona ha valorado la situación inicialmente, se forma una idea de su capacidad de controlar o afrontar el peligro, la amenaza o el desafío, impresión que se denomina valoración secundaria. Finalmente realiza una reevaluación en donde la situación anteriormente valorada adquiere otro carácter que el original, si el entorno cambia o la persona empieza a considerar la situación en forma distinta.

Rodríguez, Roque y Moleiro (1999) afirman que existen ciertas características que parecen determinar el que una situación devenga como estresante, entre ellas se destacan: el cambio o novedad, la falta de información, la no predictibilidad, incertidumbre, ambigüedad, inminencia, duración y significación para la persona, entre otras. Destacan las condiciones biológicas del organismo y la 
carencia de habilidades para hacerle frente. Se plantea, pues, que existen estresores que provienen tanto de estímulos externos a la propia persona como de aspectos internos. Los internos pueden ser estímulos de características físicas, como la sensación de malestar por mala digestión, el dolor producido por una herida, una enfermedad; o de características más cognitivas como el recuerdo de una situación desagradable, pensamientos sobre la propia inutilidad o lo difícil que puede ser el futuro inmediato, sentimientos de culpa, o ambiciones no conseguidas. Los estresores externos también pueden provenir de aspectos físicos como la temperatura ambiental extrema, el ruido o la luz intensa; o implicar aspectos cognitivos, por ejemplo: ver que dos personas hablan en voz baja y de vez en cuando lo miran y se ríen. Aunque funcionan como estresores tanto los aspectos físicos como los cognitivos, estos últimos, se dice, son más frecuentes e importantes.

También se ha planteado que las fuentes de estrés se dividen en tres tipos: sucesos vitales altamente traumáticos, tales como catástrofes naturales, terrorismo, enfermedades terminales, guerras; sucesos vitales mayores, tales como cambios laborales, matrimonio, nacimiento de un hijo, entre muchos otros; y contratiempos cotidianos, que se asume son eventos que ocurren de manera relativamente frecuente, que se caracterizan por ser irritantes, frustrantes y menores, además de que implican un cierto grado de transaccción diaria con el ambiente.

Lo estresante de una situación, desde la perspectiva de Lazarus y Folkman (1986), depende de la capacidad o incapacidad de una persona para afrontar una situación. Estos autores definieron esta capacidad como el cambio constante de los esfuerzos cognitivos y conductuales para responder a las demandas especificas de carácter externo o interno que se valoran como elementos que exceden los recursos de una persona. Consideran que la capacidad de una persona para afrontar una situación depende de numerosos factores como la salud y la energía, las creencias positivas (creer que son capaces de obtener las consecuencias deseadas), habilidad para resolver problemas, habilidades sociales, y apoyo social o sensación de aceptación. De hecho, se ha planteado que las estrategias de afrontamiento actúan como moduladores del efecto de las contrariedades cotidianas (Martínez, Rubio, Ulla, Ramos, Crespo y Hernández, 2002).

Según Rodríguez (1995) hay diferentes tipos de respuestas de afrontamiento, entre éstas se encuentran las siguientes:

- El afrontamiento centrado en la emoción: se usa para controlar la emoción, algunas veces alterando el significado de un resultado.

- El afrontamiento centrado en el problema: se usa para controlar la relación perturbada entre la persona y su circunstancia mediante la solución de problemas, la toma de decisiones o la acción directa (Lazarus y Folkman, 1986).

Por otra parte estos autores afirman que hay varias formas de afrontar el estrés:

- El afrontamiento aproximativo, que incluye todas aquellas estrategias de confrontación y enfrentamiento del problema que está generando el distrés o las emociones negativas concomitantes.

- El afrontamiento evitativo, que incluye todas aquellas estrategias cognitivas o comportamentales que permiten escapar de, o evitar, la consideración del problema o las emociones negativas concomitantes.

- El afrontamiento pasivo, cuando se omite toda acción y se permanece en situación de duda o espera.

- Afrontamiento comportamental, que implica un intento de tomar una decisión y cambiar la situación problemática, llevando a cabo conductas que la persona supone adecuadas para ello.

- El afrontamiento cognitivo, que implica un intento de tratar con los problemas mediante cogniciones. 
- Respuestas anticipatorias, que se ejecutan antes de la ocurrencia del acontecimiento estresante.

- Respuestas restaurativas, después de la ocurrencia del acontecimiento estresante.

A partir de esta concepción se han llevado a cabo numerosas investigaciones sobre estrategias de afrontamiento, y sus repercusiones en la salud. Se han investigado diversas estrategias de afrontamiento con relación a la controlabilidad de una situación (Del Valle, González, Diez, Vilda y Llorca, 2003), el tipo de estrategias más empleadas (Miro, García, Martínez-Abascal, Tortella-Feliu, Bornas, y Llabrés, 2003), relaciones entre diversas estrategias e indicadores biológicos de enfermedad (Anson, Carmel, Levenson, Bonneh, \& Maoz, 1993; McCracken, Semenchuk, \& Goetsch, 1995; Fontana \& McLaughlin, 1998; McCracken, Goetsch, \& Semenchuk, 1998; Schwartz, Peng, Lester, Daltroy \& Goldberger, 1998; Astin et al., 1999; Gallagher \& MacLachlan, 1999; Dijkstra \& De Vries, 2002), se han evaluado diversas estrategias junto con autoeficacia en relación a enfermedades (Beckham, Burker, Rice \& Talton, 1995; Blanchard, Rodgers, Courneya, Daub, \& Black, 2002; Orbell, Johnston, Rowley, Davey \& Espley, 2002), y se ha planteado que existe relación entre tipos de personalidad y afrontamiento (Claridge, 1983; Pervin, 1998; Sarafino, 1998; Ramírez, Esteve y López, 2001; Ferguson, 2002).

\section{Propuesta conceptual}

Bajo una perspectiva naturalista y partiendo de criterios funcionales, se pueden hacer algunas consideraciones en relación al fenómeno que se aborda bajo la perspectiva estrés/afrontamiento. Para empezar hay que señalar que este abordaje se caracteriza por una aproximación dualista. Las implicaciones de considerar que existen procesos mentales y ocultos responsables del comportamiento, han sido analizadas con detalle por otros autores (Ryle, 1949; Ribes, 1982, Turbayne, 1982; Ribes y López, 1985; Ribes, 2001), por lo que en este trabajo nos vamos a enfocar en otras consideraciones; partimos de que un análisis con criterios funcionales permitiría una mejor comprensión del fenómeno. Para ello conviene desglosar tres aspectos: a) la naturaleza funcional de la situación, b) los niveles de aptitud funcional en el comportamiento y c) dimensiones del comportamiento.

\section{La naturaleza funcional de la situación}

Desde una perspectiva no dualista, la llamada reacción de estrés está absolutamente vinculada con las contingencias ambientales que son las que afectan de manera sistemática las reacciones inmunitarias del organismo, especialmente la referida a la producción de linfocitos y esta influencia parece ser mediada a través de las reacciones de los sistemas pituitario y neurovegetativo (Moberg, 1985). Dicha reacción parece adoptar diversidad de formas más o menos específicas, dependiendo de la intensidad y duración de las condiciones de estímulo, así como de la participación diferencial de distintos subsistemas biológicos. Por ello, la identificación de circunstancias ambientales sistemáticamente vinculadas a reacciones de estrés, específicas o generalizadas, parece constituir una estrategia adecuada para examinar la forma en que el ambiente afecta a los estados biológicos relacionados con la vulnerabilidad a condiciones o agentes patógenos (Ribes, 1990a).

La identificación de circunstancias ambientales sistemáticamente vinculadas a reacciones de estrés debe trascender los criterios morfológicos y centrarse en la naturaleza de las relaciones funcionales que las caracterizan. Las situaciones que, desde otras perspectivas se entienden como estresores, no son otra cosa que arreglos contingenciales o campos de contingencias, es decir, relaciones condicionales entre las acciones de un individuo y las acciones de otro (s), así como de los objetos y condiciones ambientales potencial y actualmente efectivos (Ribes, 1990b). Entender y estudiar la naturaleza funcional de una situación implica la descripción de estas relaciones de condicionalidad, así como la de sus elementos definitorios. Una situación, por ejemplo, puede presentar demandas que solamente pueden satisfacerse con una respuesta que es la que produce consecuencias favorables; en otras situaciones puede haber dos o más demandas incompatibles y la persona debe elegir qué hacer; hay situaciones cuyos elementos definitorios están dados porque las demandas presentan variaciones 
a las que hay que ajustarse; o arreglos en donde los factores de contexto tienen una mayor influencia. El empleo de criterios funcionales permite esclarecer la naturaleza funcional de un conjunto de situaciones. La descripción que tradicionalmente se hace de las situaciones consideradas productoras de estrés se basa en criterios morfológicos e incluye, desde estímulos puntuales, hasta respuestas de la propia persona, pasando por situaciones complejas compuestas de diversos elementos. Se mencionan, por ejemplo, estímulos tales como la luz intensa, o el ruido; situaciones como la pérdida de un ser querido; condiciones biológicas como enfermedades; y la propia conducta de la persona como pensamientos o recuerdos. Este abordaje impide, por un lado, delimitar eventos del ambiente y eventos del comportamiento, y por otro, entender las relaciones de condicionalidad que caracterizan las diversas situaciones; además, una descripción morfológica de cada situación específica resulta una tarea inagotable.

Con el propósito de ilustrar el empleo de criterios funcionales en la descripción de situaciones, vamos a citar el trabajo de Ribes (1990a) con relación a lo que denomina 'estilos interactivos'. Para estudiar, desde una perspectiva interconductual, lo que tradicionalmente se aborda como personalidad, Ribes (op. cit.) definió un conjunto de arreglos contingenciales o situaciones genéricas de manera funcional. Elaboró una taxonomía que incluye 12 arreglos. Uno de ellos se denomina tolerancia a la frustración, Ribes (op. cit.) lo describe como: toda aquella situación específica en donde una persona desarrolla alguna actividad y de manera no advertida lo que obtiene como consecuencia de su conducta:
a) es menor que lo esperado,
b) se demora,
c) no se otorga,
d) se le retira sin razón,
e) se le impide desarrollar la conducta.

Esta definición describe relaciones condicionales entre lo que hace una persona y acontecimientos específicos del ambiente. Los elementos definitorios tienen que ver, por un lado, con la ausencia de señales o advertencias y, por otro, con la magnitud, retiro o demora de las consecuencias. En ambientes naturales este arreglo genérico encuentra diversos ejemplos en situaciones específicas. Pensemos en una persona que lleva a cabo un trabajo que acostumbran pagarle con cierta cantidad y a la que sin previo aviso le pagan menos, o en alguien que está trabajando utilizando un aparato electrónico que se sobrecalienta y provoca una falla general de electricidad que le impide continuar trabajando, o en alguien más que presta dinero a un amigo quien promete devolverlo en una semana y no lo hace. La identificación funcional de situaciones genéricas permite describir relaciones de condicionalidad comunes a muchas situaciones específicas, y por ende, una exploración más sistemática de las mismas.

Con este tipo de aproximación un factor a considerar es la identificación de demandas de logro. Si una situación requiere que la persona emita una respuesta correcta, ajuste su comportamiento a ciertas circunstancias, produzca un resultado específico o resuelva un problema, el tipo de arreglo contingencial es cerrado; por el contrario, si la situación no presenta demandas de logro, se trata de un arreglo contingencial abierto. La naturaleza cerrada o abierta de una situación es uno de los elementos que permite definir el tipo de comportamiento que se estudia. En el primer caso, la persona responde de acuerdo a su capacidad y ello implica el estudio de competencias. En el segundo caso, la persona responde fundamentalmente de acuerdo a su historia interactiva, lo que permite el estudio de estilos interactivos (Ribes, 1990a).

Ribes (1990a) ha definido el término 'competencia' como la posesión de un conjunto de destrezas y habilidades en respuesta a un requerimiento o demanda en una situación particular. Este término especifica qué destrezas o habilidades se requieren para interactuar con las exigencias y oportunidades del ambiente. Hablar de competencia es hablar de que un individuo es capaz para algo, por ello se requiere que exista una correspondencia entre las propiedades conductuales del organismo (la morfología de la 
conducta) y la morfología (fisicoquímica, ecológica, convencional) de los estímulos ambientales, la cual considera una extensa gama de todas las posibles interacciones entre un individuo y su ambiente (Corral, 2003). Las competencias no equivalen a respuestas o habilidades, sino que se identifican con disposiciones, esto es, con la propensión a comportarse de acuerdo a las exigencias del ambiente, por ello 'competencia' es una categoría disposicional, no designa una forma concreta de responder, ni corresponde con el estudio de variables u ocurrencias, sino con el de disponibilidad interactiva (Ribes y López, 1985). Como lo define Ryle (1949), 'competencia' es un factor disposicional que tiene que ver con logro. No equivale al concepto de habilidad ni se identifica con una forma concreta de comportamiento, sino que designa un conjunto de habilidades en potencia, y es por ello que el término se emplea en función de la historia de un individuo (Ribes, 1990a).

Los estilos interactivos, por su parte, constituyen la manera consistente e idiosincrásica en la que un individuo interactúa con una situación en la que no existen criterios claros o explícitos respecto al cómo debe comportarse; es decir, en donde no existen criterios de logro o demandas explícitas (Ribes, 1990a). Esta categoría también es una categoría disposicional. No designa respuestas particulares u ocurrencias, sino modos o maneras de responder a circunstancias ambientales específicas. Los estilos interactivos se prevén como consistentes en el tiempo y en distintas situaciones específicas que pueden ser caracterizadas por el mismo arreglo contingencial, es decir, por el mismo tipo de relación funcional entre objetos, personas, circunstancias y acontecimientos.

Como señalábamos, Ribes (1990a) tipificó situaciones o arreglos contingenciales para estudiar modos consistentes y característicos de cada persona, es decir, estilos interactivos. Los arreglos son abiertos, esto es, sin ningún criterio de logro o efectividad; y planteó, con base en una serie de estudios (Moberg, 1985; Kelley, 1985; Levine, 1985), que seis de estos arreglos parecen corresponder parcial o totalmente, a las características funcionales bajo las cuales se produce el estrés, mientras que los otros seis comprenden contingencias vinculadas más bien a efectividad o a condiciones que la delimitan. Se supone que estos últimos influyen preponderantemente sobre las competencias funcionales efectivas en el ámbito de interacción del individuo. Las situaciones o arreglos correspondientes a la reacción de estrés se caracterizan por relaciones de condicionalidad con respecto a: toma de decisiones, frustración, reducción de conflicto, ambigüedad, tendencia al riesgo, e impulsividad/no impulsividad; cada uno de estos ellos presenta condiciones definitorias que pueden ir desde la incompatibilidad o imprevisibilidad de circunstancias ambientales, hasta la regulación del comportamiento por disposiciones personales tales como tendencias o condiciones emocionales, que pueden ser incongruentes con las demandas de la situación. Un dato a considerar es que ninguna de estas situaciones demanda una respuesta específica, es decir, correcta. Por otro lado, los arreglos o situaciones que influyen básicamente en la capacidad personal tienen que ver con: persistencia o logro, flexibilidad al cambio, trasgresión, curiosidad, dependencia de señales y responsividad a nuevas contingencias.; algunos de estos arreglos pueden incluir demandas que exigen respuestas específicas, y en esa medida, se pueden adaptar para estudiar competencias.

No es propósito de este trabajo describir la taxonomía de estilos interactivos propuesta por Ribes (1990a), aunque es importante considerar que esta manera de proceder ilustra la forma de aproximación, que en primera instancia, aquí se propone para abordar el fenómeno de estrés.

En la aproximación cognoscitiva al fenómeno estrés/afrontamiento no se lleva a cabo ninguna distinción entre campos de contingencias abiertos y campos cerrados, de manera que el concepto de "afrontamiento" no delimita tipos distintivos de comportamiento y, en esa medida, margina, tanto parámetros importantes de las propias situaciones como las características distintivas de cada comportamiento.

\section{Niveles de aptitud funcional en el comportamiento}

Al proponer el empleo de criterios funcionales para estudiar los fenómenos abordados bajo el concepto estrés/afrontamiento, además de considerar la naturaleza funcional de la situación, hay que entender que las personas se comportan con distintos niveles de complejidad funcional. Ribes y López (1985) sostienen que el comportamiento puede tener diversos niveles de complejidad. 
Elaboraron una taxonomía funcional que define estos niveles inclusivos de complejidad creciente. Con base en esta taxonomía, Ribes (1990a) delimitó cuatro niveles funcionales en que pueden tener lugar las competencias efectivas en una situación: a) situacional no instrumental, b) situacional instrumental, c) extrasituacional y d) transituacional. La taxonomía parte de lo que se consideran niveles de desligamiento funcional.

El nivel situacional no instrumental representa el proceso más simple de aptitud funcional, y el transituacional el más complejo. En el nivel situacional no instrumental no hay un desligamiento funcional. La persona responde a los elementos presentes en una situación a través de reacciones diferenciales ante los objetos, personas y acontecimientos presentes, sin alterar sus propiedades funcionales. Los procesos preceptúales, las reacciones emocionales o comportamientos de seguimiento de instrucciones, son ejemplos de este tipo de interacciones.

En el segundo proceso (nivel situacional instrumental) tampoco hay desligamiento funcional. La persona también se comporta en relación a objetos, personas y circunstancias presentes en una situación, aunque introduce cambios en el ambiente como efecto de su conducta, lo cual a su vez, afecta favorable o desfavorablemente su propio comportamiento. Ejemplos de este proceso son: preparar un platillo, reparar un mueble roto, acudir al médico ante síntomas de alguna enfermedad, pedirle a alguien que abra una ventana, etcétera.

Los siguientes dos niveles de aptitud funcional: extrasituacional y transituacional, se caracterizan por implicar procesos sustitutivos de comportamiento. Ribes y López (1985) definieron la conducta sustitutiva como conducta convencional que transforma, de manera lingüística, las contingencias objetivas de una situación. Ribes (1990a) comenta que dicha "transformación" alude a la capacidad de desligar conductas particulares de su correspondencia funcional con las contingencias físicas presentes en una situación para ligarlas a circunstancias no presentes en la situación actual. El carácter convencional del comportamiento humano es el que permite este desligamiento de las propiedades físico-químicas de los objetos y acontecimientos presentes en el aquí y el ahora. Las personas somos capaces de recordar, opinar, juzgar, planear, o reflexionar, al margen de circunstancias específicas presentes. Ribes (1990b) habla de dos tipos de desligamiento funcional, el que corresponde a un proceso extrasituacional, en donde la persona actúa frente a los elementos y contingencias presentes como si tuvieran las propiedades de otros elementos o contingencias, o el relativo a un proceso transituacional, en donde el individuo responde a las contingencias situacionales presentes en términos de las propiedades lingüísticas de su conducta, de modo que su comportamiento se vuelve independiente de cualquier situación específica. El primer caso puede ilustrarse con el comportamiento de una persona que se pone tensa una semana antes de una cita para conseguir empleo, o de alguien que piensa continuamente que algo va a salir mal cuando se le pide organizar una reunión, o de una persona que recuerda un acontecimiento. En estos casos las personas se comportan como si estuvieran relacionándose con otras circunstancias, pasadas, distantes o futuras, y las contingencias transformadas afectan al individuo como si estuviera presente en tales situaciones. El segundo caso se puede caracterizar como el "hablar acerca del hablar" (Ribes, 1990b, p. 191). Ejemplos de este proceso son escribir un ensayo, teorizar, componer una obra musical, hacer críticas literarias, o comportarse de acuerdo a teorías o filosofías de vida. Aquí, el comportamiento está regulado por la propia conducta lingüística.

Al estudiar el comportamiento es importante distinguir su nivel funcional, en tanto, implica procesos diferentes y, específicamente si el comportamiento a estudiar se relaciona con competencias, hay que considerar que su nivel de aptitud debe guardar una correspondencia funcional con lo que la propia situación demanda, esto es, no hay un nivel de aptitud funcional "mejor" que otro, el responder de una manera competente en una situación va a depender de lo que la propia situación requiere. Así, por ejemplo, si una situación determinada requiere la solución de un problema matemático, y para ello hay que responder empleando una lógica matemática, el nivel de aptitud demandado es un nivel transituacional; la persona tiene que trascender las condiciones presentes de la situación que tiene lugar en la forma de objetos y acontecimientos presentes y responder a teorías y conocimientos especializados que no tienen que ver las circunstancias concretas en las que se encuentra. Responder 
a una situación como ésta, solamente con ciertos sentimientos de angustia o enojo, por ejemplo, sería enfrentar la situación de manera incompetente.

Pero no todo tipo de situación demanda un nivel de respuesta transituacional, si por ejemplo, un médico le pide a su paciente que lleve un registro diario de su presión arterial y lo cita para el siguiente mes; la situación demanda un nivel competencial extrasituacional. La persona debe tomarse la presión cotidianamente respondiendo como si estuviera con el médico.

\section{Dimensiones del comportamiento}

Además de los niveles de aptitud funcional, el comportamiento puede estudiarse considerando distintas dimensiones. Kantor (1926) ha planteado que el comportamiento puede ser efectivo o afectivo. Ribes (1990b) y Corral (1997) agregan una dimensión valorativa.

La dimensión efectiva se refiere a las prácticas de los individuos en términos de lo que hacen y dicen al relacionarse con su ambiente, produciendo cambios en objetos, acontecimientos o conducta de otras personas. A esta dimensión se le llama efectiva porque produce efectos. Como la dimensión efectiva del comportamiento se refiere a aquellas formas de responder que generan cambios en el ambiente, podemos identificarla en diversos niveles de aptitud funcional: instrumental, extrasituacional o transituacional.

La dimensión afectiva, por su parte, se refiere a aquellos componentes del comportamiento o a aquellos comportamientos que solamente afectan a la propia persona que los emite y que no producen cambios en el ambiente. Las conductas de orientación, de atención, procesos perceptuales, y en general, reacciones que corresponden al lenguaje de los sentimientos, ilustran este tipo de comportamiento. Por ejemplo, cuando un individuo observa un paisaje, percibe un objeto específico, siente angustia, alegría, o incomodidad, su comportamiento no afecta al ambiente, sino que los cambios solamente se producen en él mismo. El nivel situacional no instrumental, en tanto proceso de comportamiento que no genera cambios en el ambiente (inefectivo) alude a comportamientos puramente afectivos. Vale la pena aclarar que el comportamiento afectivo puede formar parte de un patrón conductual más complejo, de manera que una persona puede hacer algo y generar cambios en objetos, circunstancias o comportamiento de otros, y al mismo tiempo percibir, atender o sentir ciertas cosas. El comportamiento afectivo no provoca sus acciones ni es consecuencia de las mismas, sino que es parte del modo en que se relaciona con su ambiente. Una persona, por ejemplo, puede discutir con otra acerca de algo (dimensión efectiva) y estar sintiendo enojo (dimensión afectiva). Ninguna dimensión actúa como agente causal de la otra.

Por último está la dimensión valorativa. Esta dimensión solamente incluye procesos sustitutivos. Los juicios, las evaluaciones o las valoraciones no son un comportamiento distinto al de las acciones, sino una dimensión de las mismas, y corresponden a niveles extrasituacionales o transituacionales. Las creencias y valoraciones son comportamiento sustitutivo que tiene una función reguladora de otros comportamientos propios (Carpio, Pacheco, Hernández y Flores, 1995). Se refiere, en términos generales, a las creencias y éstas incluyen aspectos evaluativos, atributivos, informativos y culturales. Esta dimensión refiere prácticas característicamente humanas vinculadas con la cultura, descritas desde una perspectiva psicológica. Las creencias, cuando se dan en un nivel extrasituacional, permiten que una persona se ajuste a una situación presente interactuando como lo haría en otras situaciones; mientras que cuando se dan en un nivel transituacional permiten al individuo relacionarse con otros comportamientos o productos convencionales. En cualquiera de los dos casos, la persona se abstrae de las condiciones estimulantes presentes en una situación concreta. El individuo sustituye las morfologías físico-químicas u organísmicas de los elementos que conforman una situación, por morfologías convencionales. Las creencias no describen la realidad, sino que constituyen un comportamiento que se construye a partir de ella. Como comportamiento convencional, no corresponden a situaciones específicas, y muchas veces, ni al propio comportamiento efectivo (Corral, 1997). Las creencias presentan correspondencias convencionales, con otras situaciones o campos de contingencias (pasadas, futuras o distantes), o con otros comportamientos lingüísticos (ideales, filosofías o preceptos). 
Estos conceptos no refieren procesos mentales, sino características funcionales del comportamiento convencional, tampoco refieren procesos causa-efecto, o una doble acción: creer y hacer, o creer y decir; sino procesos complejos de comportamiento.

Si consideramos los criterios propuestos, las personas pueden reaccionar con estrés en múltiples situaciones. Una descripción morfológica de situaciones específicas es inagotable, y conceptualmente limitada, en tanto las reacciones de estrés no necesariamente corresponden a ciertas situaciones. La misma situación puede generar estas reacciones en un individuo y no generarlas en otro. $\mathrm{Si}$, por otra parte, describimos el comportamiento de cada persona, con criterios morfológicos, la tarea también supera cualquier esfuerzo. La manera de relacionarse de cada individuo con situaciones específicas es singular. El estudio de este fenómeno, con fines aplicados, debe partir del individuo.

Considerando lo anterior proponemos abordar el fenómeno dirigiendo las investigaciones hacia la creación de una taxonomía de situaciones genéricas que permita explorar diversos aspectos del comportamiento de cada persona. Aquí se propone emplear, en primera instancia, la taxonomía preliminar generada por Ribes (1990a) sobre estilos interactivos. Con base en los 12 arreglos contingenciales se pueden diseñar situaciones que impliquen campos de contingencias abiertos, o se pueden incluir, para algunos de ellos, criterios de logro para que estos campos sean cerrados. En un segundo momento se pueden agregar elementos o variar las condiciones parámetricas de cada situación. Por ejemplo, se pueden agregar elementos disposicionales de contexto, Posteriormente, hay que configurar un mayor número de situaciones genéricas, a partir de la identificación de situaciones específicas, y ampliar con ello la taxonomía.

Las situaciones diseñadas servirían para estudiar el comportamiento individual. Como señalábamos, las formas de responder en una situación pueden ser muy diversas dada la capacidad humana de desligamiento funcional. Si las personas respondiéramos solamente a los elementos presentes en forma concreta en una situación, la taxonomía a desarrollar debería clasificar las situaciones que producen, reacciones de estrés y las que no; sin embargo, las llamadas reacciones de estrés pueden presentarse en situaciones que objetivamente no están vinculadas a ello, mediadas por comportamiento extrasituacional o transituacional; por ello, es relevante identificar un conjunto de situaciones genéricas que, en una primera instancia, permitan delimitar fenómenos generales, en principio, cuándo estudiamos competencias y cuándo estilos interactivos.

A partir de ello se pueden estudiar diversos elementos en el comportamiento:

-Cuando la situación demanda competencias:

¿Posee la persona las competencias demandadas?

¿Ante estas situaciones, presenta reacciones de estrés?

Si presenta reacciones de estrés, ¿éstas están reguladas los elementos presentes en la situación o por procesos sustitutivos?

¿Si están reguladas por creencias, éstas pertenecen a un sistema valorativo general?

-Cuando la situación no demanda competencias:

¿La persona presenta reacciones de estrés?

¿La situación está relacionada objetivamente con estas reacciones?

¿La persona responde de manera consistente a este tipo de situaciones?

¿Su manera de responder está regulada por la propia situación o por procesos sustitutivos?

¿Si su comportamiento está regulado por creencias, éstas pertenecen a un sistema valorativo general?

Estas son algunas de las preguntas que podrían formularse, aunque hay mucho por investigar. Las metodologías para aproximarse a esta propuesta pueden ser diversas y su utilidad dependerá fundamentalmente del planteamiento de preguntas pertinentes. Para la identificación de un conjunto 
de situaciones específicas que permitan desarrollar una taxonomía de situaciones genéricas se puede recurrir a la elaboración y aplicación de instrumentos de autoreporte, o a la observación sistemática. El comportamiento de los individuos en estas situaciones se puede estudiar, en sus dimensiones efectiva, afectiva y valorativa, también puede estudiarse de manera experimental o empleando instrumentos de reporte verbal. Se pueden emplear, como se ha venido haciendo, instrumentos para medir indicadores biológicos de las reacciones de estrés, con el propósito de avaluar el comportamiento afectivo, y específicamente, para tener una mejor comprensión de la dimensión valorativa, conviene desarrollar modelos que permitan identificar la regulación afectiva y efectiva por procesos sustitutivos, así como la dependencia de creencias particulares a sistemas valorativos generales. Finalmente, se puede pensar en relacionar comportamientos específicos con el estado de salud/enfermedad.

Una aproximación de esta naturaleza permitiría abordar el fenómeno de una manera naturalista y sistematizar su estudio con criterios funcionales.

\section{Referencias}

Anson, O., Carmel, S., Levenson, A., Bonneh, D., \& Maoz, B. (1993). Coping with recent life events: the interplay of personal and collective resources. Behavioral Medicine, 18, 4, 159-166.

Astin, J. A., Anton-Culver, H., Schwartz, C. E., Shapiro, D. H. Jr., McQuade, J., Breuer, A. M., Taylor, T. H., Lee, H., \& Kurosaki, T. (1999). Sense of control and adjustment to breast cancer: the importance of balancing control coping styles. Behavioral Medicine, 25, 3, 101-109.

Beckham, J. C., Burker, E. J., Rice, J. R., \& Talton, S. L. (1995). Patient predictors of caregiver burden, optimism, and pessimism in rheumatoid arthritis. Behavioral Medicine, 20, 4, 171-178.

Blanchard, C. M., Rodgers, W. M., Courneya, K. S., Daub, B., \& Black, B. (2002). Self-efficacy and mood in cardiac rehabilitation: should gender be considered? Behavioral Medicine, 27, 4, 149-160.

Brannon, L. y Feist, J. (2001). Definición y medición del estrés. Psicología de la salud. Madrid: Thompson Learning.

Carpio, C., Pacheco, V., Hernández, R., y Flores, C. (1995). Creencias, criterios y desarrollo psicológico. Acta Comportamentalia, 3, 1, 89-98.

Cassidy, T. (1999). Stress, cognition and health. London: Routledge.

Claridge, G. (1983). Relaciones psicosomáticas en la enfermedad física. En: H J. Eysenck. Manual de psicología anormal. México: El Manual Moderno.

Corral, V. (1997). Disposiciones psicológicas: un análisis de las propensiones, capacidades y tendencias del comportamiento. México: UNISON.

Corral, V. (2003). ¿Mapas cognoscitivos o competencias ambientales? En: E. Díaz-González y M. L. Rodríguez (compiladores). Perspectivas sobre el Cognoscitivismo en Psicología. México: UNAM, Facultad de Estudios Superiores Iztacala.

Del Valle, C., González, M., Diez, M., Vilda, B y Llorca, G. (2003). Estudio de estrategias de afrontamiento a través de las diferentes fases del proceso de trasplante de medula ósea antólogo. Psicología conductual 11, 2, 293-306.

Dijkstra, A. \& De Vries, H. (2002). Do self-help interventions in health education lead to cognitive changes, and do cognitive changes lead to behavioural change? British Journal of Health Psychology, 6, 2, 121-134.

Ferguson, E. (2002). Personality and coping traits: A joint factor analysis. British Journal of Health Psychology, 6, 4, 311-325. 
Fontana, A. \& McLaughlin, M. (1998). Coping and appraisal of daily stressors predict heart rate and blood pressure levels in young women. Behavioral Medicine, 24, 1, 5-16.

Gallagher, P., \& MacLachlan, M. (1999). Psychological adjustment and coping in adults with prosthetic limbs. Behavioral Medicine, 25, 3, 117-124.

Kantor, J. R. (1926). Principles of Psychology. Chicago: Principia Press.

Kelley, K. W. (1985). Inmunological consequences of changing environmental stimuli. En G. P. Moberg (Ed.). Animal stress. Bethesda: American Physiological Society.

Lazarus, R. S. y Folkman, S. (1986). Estrés y procesos cognitivos. Barcelona: Martínez Roca.

Levine, S. (1985). A definition of stress? En G. P. Moberg (Ed.). Animal stress. Bethesda: American Physiological Society.

Martínez, A., Rubio, V., Ulla, S., Ramos, J., Crespo, N. y Hernández, J. (2002). Diferencias individuales en la influencia del estrés sobre la sintomatología asmática. Psicología Conductual, 10,1 ,

McCracken, L. M., Semenchuk, E. M., \& Goetsch, V. L. (1995). Cross-sectional and longitudinal analysis of coping responses and health status in persons with systemic lupus erythematosus. Behavioral Medicine, 20, 4, 179-187.

McCracken, L. M., Goetsch, V. L., \& Semenchuk, E. M. (1998). Coping with pain produced by physical activity in persons with chronic low back pain: immediate assessment following a specific pain event. Behavioral Medicine. 24, 1, 29-34.

Miró, J., García, G., Martínez-Abascal, A., Tortella-Feliu, M., Bornas, X., y Llabrés, J. (2003). Estilo de afrontamiento y resultados del tratamiento de exposición en sujetos con fobia a volar. Revista Internacional de Psicología Clínica y de la Salud. 3, 3, 477-487.

Moberg, G. P. (1985). Animal stress. Bethesda: American Physiological Society.

Orbell, S., Johnston, M., Rowley, D., Davey, P., \& Espley, A. (2002). Self-efficacy and goal importance in the prediction of physical disability in people following hospitalization: A prospective study. British Journal of Health Psychology, 6, 1, 25-40.

Pervin, L. A. (1998). La ciencia de la personalidad. España: McGraw-Hill.

Ramírez, C., Esteve, R. y López, A. (2001). Neuroticismo, afrontamiento y dolor crónico. Anales de psicología, 17,1, 129-137.

Ribes, E. (1982). El Conductismo: Reflexiones críticas. Barcelona: Fontanella.

Ribes, E. (1990a). El comportamiento y la prevención de la enfermedad. Psicología y salud: un análisis conceptual. Barcelona: Martínez Roca.

Ribes, E. (1990 b). Psicología General. México: Trillas.

Ribes, E. y López, F. (1985). Teoría de la conducta: un análisis de campo y paramétrico. México: Trillas.

Ribes, E. (2001). Los conceptos cognoscitivos y el problema de la observabilidad. Acta Comportamentalia, 9, Monográfico, 9-19.

Rodríguez, J. (1995). Estrés psicosocial y su afrontamiento. España: Psicología Social de la Salud.

Rodríguez, R, Roque, Y., y Moleiro, O. (1999). Estrés laboral, consideraciones sobre sus características y formas de afrontamiento. Universidad Central "Marta Abreu " de Las Villas.

Ryle, G. (1949). The concept of mind. N. Y: Barnes and Noble.

Sarafino, E. P. (1998). Health Psychology. Biopsychosocial interactions. USA : John Wiley \& Sons, INC. 
Schwartz, C. E., Peng, C. K., Lester, N., Daltroy, L. H., \& Goldberger, A. L. (1998). Self-reported coping behavior in health and disease: assessment with a card sort game. Behavioral Medicine, 24, 2, 81-88.

Seyle, H. (1936). A syndrome produced by diverse noxious agents. Nature, 2, 132-138.

Turbayne, C. M. (1982). El mito de la metáfora. México: Fondo de Cultura Económica. 